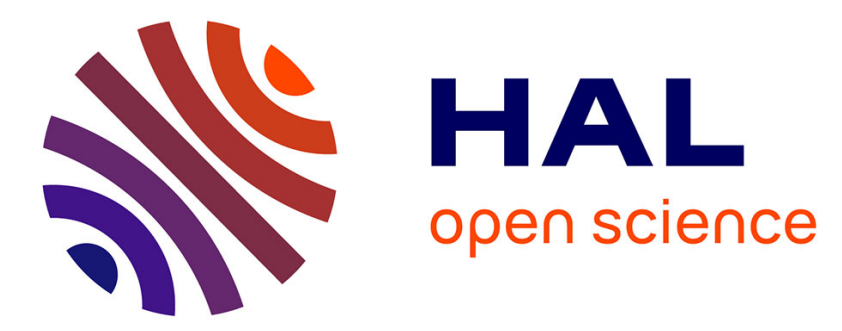

\title{
Trans-to-cis photoisomerization of cyclocurcumin in different environments rationalized by computational photochemistry
}

Marco Marazzi, Antonio Francés-Monerris, Maxime Mourer, Andreea Pasc, Antonio Monari

\section{To cite this version:}

Marco Marazzi, Antonio Francés-Monerris, Maxime Mourer, Andreea Pasc, Antonio Monari. Transto-cis photoisomerization of cyclocurcumin in different environments rationalized by computational photochemistry. Physical Chemistry Chemical Physics, 2020, 22, pp.4749-4757. 10.1039/c9cp06565b . hal-02476342

\section{HAL Id: hal-02476342 \\ https://hal.univ-lorraine.fr/hal-02476342}

Submitted on 12 Feb 2020

HAL is a multi-disciplinary open access archive for the deposit and dissemination of scientific research documents, whether they are published or not. The documents may come from teaching and research institutions in France or abroad, or from public or private research centers.
L'archive ouverte pluridisciplinaire HAL, est destinée au dépôt et à la diffusion de documents scientifiques de niveau recherche, publiés ou non, émanant des établissements d'enseignement et de recherche français ou étrangers, des laboratoires publics ou privés. 


\title{
ARTICLE
}

\section{Trans-to-cis photoisomerization of cyclocurcumin in different environments rationalized by computational photochemistry. ${ }^{+}$}

Received 00th January 20xx, Accepted 00th January 20xx DOI: $10.1039 / \times 0 \times x 00000 x$

\author{
Marco Marazzi, *a,b Antonio Francés-Monerris, ${ }^{c, d}$ Maxime Mourer, ${ }^{\text {e }}$ Andreea Pasc ${ }^{\mathrm{e}}$ and Antonio \\ Monari*c
}

\begin{abstract}
Cyclocurcumin is a turmeric component that attracted much less attention compared to the well known curcumin. In spite of the less deep charcaterization of its properties, cyclocurcumin has shown promising anticancer effects when used in combination with curcumin. Especially, due to its peculiar molecular structure, cyclocurcumin can be regarded as an almost ideal photoswitch, whose capabilities can also be exploited for relevant biological applications. Here, by means of state-ofthe-art computational methods for electronic excited-state calculations (TD-DFT, MS-CASPT2, XMS-CASPT2) we analyze in detail the absorption and photoisomerization pathways leading from the more stable trans isomer to the cis one. The different molecular surroundings, taken into account by means of electrostatic solvent effect and compared with available experimental data, have been found to be critical in describing the fate of irradiated cyclocurcumin: while in non-polar environments an excited state barrier prevents photoisomerization and favours fluorescence, in polar solvents an almost barrierless path results in a strikingly decrease of fluorescence, opening the way toward a crossing region with the ground state and thus funneling the photoproduction of the cis isomer.
\end{abstract}

\section{Introduction}

The extracts of Curcuma longa, also known as turmeric, ${ }^{1,2}$ have been widely used for centuries both as spices and food colouring agents. ${ }^{3}$ They are characterized by a rather intense and typical yellow colour due to the presence of conjugated polyphenol backbone characteristic of curcuminoid. While the most abundant component is the curcumin and their closely related derivatives, in the nineties an additional component known as cyclocurcumin was isolated and its structure was reported. In addition to its use in food industry, turmeric derivatives, and curcumin in particular, have started attracting considerable interest due to their biological and pharmaceutical properties. ${ }^{4-10}$ Indeed, the antioxidant, antibacterial and antiviral properties of curcumin have been unequivocally demonstrated. ${ }^{10}$ Moreover, its absorption in the visible range makes turmeric extracts, including curcumin, a most valuable candidate for its use in photodynamic therapy (PDT), ${ }^{11}$ or for light-assisted food decontamination, ${ }^{12,13}$ despite its rather

\footnotetext{
a. Department of analytical chemistry, physical chemistry and chemical engineering Universidad de Alcalá, Ctra. Madrid-Barcelona, Km 33,600, E-28805, Alcalá de Henares (Madrid), Spain.

b. Chemical Research Institute "Andrés M. del Rio" (IQAR), Universidad de Alcalá, Ctra. Madrid-Barcelona, Km 33,600, E-28805, Alcalá de Henares (Madrid), Spain. c. Université de Lorraine and CNRS, LPCT UMR-7019, F-5400 Nancy, France.

d. Departement de Quimica Fisica, Universitat de Valencia, 46100 Burjassot, Spain.

e. Université de Lorraine and CNRS, L2CM UMR 7053, F-54000 Nancy, France.

tElectronic Supplementary Information (ESI) available: multistate (MS)-CASPT2 benchmark with different active spaces; extended multistate (XMS)-CASPT2 energy pathways; simulated and experimental absorption spectrum of cis-cyclocurcumin Cartesian coordinates (in Ångström) and 3D structures of the most relevant geometries along the photoreaction; spin-flip TD-DFT potential energy surfaces. See DOI: $10.1039 / x 0 \times x 00000 x$
}

limited solubility in aqueous environment, that usually requires the development of appropriate drug delivering strategies. ${ }^{14-19}$ Curcumin-primed exosomes have also been used in vivo to treat neuro-degenerative disorder in mice showing extremely promising potentiality in alleviating the symptoms of Alzheimer's disease. ${ }^{20}$ The photophysics of curcumin and its complex modulation due to the interplay between the packing in liposomes, and the modulation of keto-enol tautomerism has also been recently addressed by a combination of spectroscopic and computational techniques. ${ }^{21}$

In contrast, the biological properties of cyclocurcumin are much less characterized than the ones of its parent compound, and its use is much more limited. ${ }^{22,23}$ Indeed, a more limited anticancer activity compared to curcumin has been recognized for cyclocurcumin against the proliferation of brain cancer cell lines. ${ }^{24,25}$ Nevertheless, the antiproliferative activity of curcuminoids increases sensibly when they are applied in combination, suggesting a synergic effect.

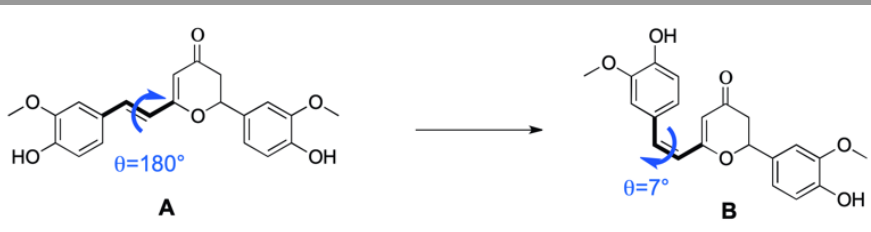

Figure 1) Molecular structure of trans- (A) and cis- (B) cyclocurcumin isomers. Cyclocurcumin photoisomerization of the double bond around the dihedral angle $\theta$ is envisaged to be responsible for the process.

As it can be inferred from Figure 1, cyclocurcumin is characterized by the presence of a carbon-carbon double bond that gives rise to the coexistence of cis and trans isomers. As it 
is evident from sterical considerations, the trans isomer is the thermodynamically favoured one and it is indeed the one present in the natural extract. Compounds presenting isomerizable double bonds within rather extended $\pi$ conjugated backbones, such as carotenoids and retinal,26-32 or azobenzene $\mathrm{e}^{33-35}$ and stilbene $\mathrm{3}^{3-38}$ are regarded as paradigmatic molecular photoswitches, 39,40 i.e. compounds able to undergo photoisomerization after the absorption of visible light. The study of natural photoswitches has been extremely active and has allowed to important breakthroughs in the last years, also allowing the engineering of bio-inspired synthetic photoswitches. ${ }^{41,42}$ In this respect, cyclocurcumin presents an almost optimal molecular structure, with the presence of only one isomerizable double bond, despite a non-negligible overlap between the absorption spectra of the two isomers. The presence of a single isomerizable bond is highly desirable, since it allows a fine control of the photoswitching activity between two defined states (i.e. trans and cis isomers, avoiding the complexity due to a higher number of states). However, and despite its attractiveness, its photophysical properties have been only marginally addressed in the literature. In particular, Adhikary et $a l .{ }^{43}$ have reported the influence of the molecular environment, and specifically of the polarity of the solvent, on the fluorescence quantum yield of cyclocurcumin. By low temperature fluorescence decay analysis the authors have also concluded that cyclocurcumin is most likely performing transto-cis photoisomerization in polar solvents, only pointing to a strong dependence on the polarity and the viscosity of the medium. ${ }^{43}$ However, the fine molecular and electronic factors hiding behind the environmental dependence of the photoisomerization efficiency, are not rationalized, yet. In particular, no proper characterization of the potential energy surfaces (PES) of the different excited states involved in the
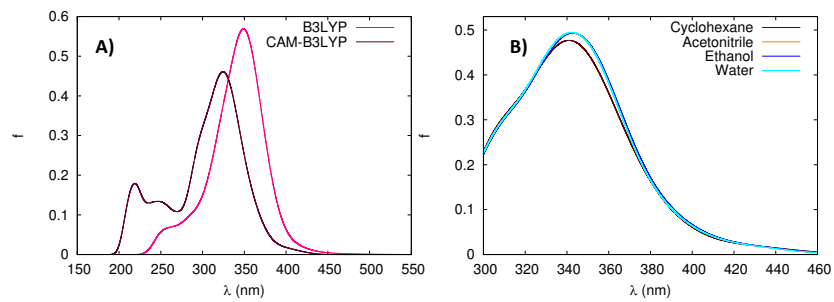

process, and their modulation by the molecular surroundings has been provided.

Figure 2) TD-DFT calculated absorption spectrum of cyclocurcumin in gas phase (A) and in different solvents (B). A comparison between B3LYP and CAM-B3LYP
functionals is shown for the gas phase spectrum, while the CAM-B3LYP functional was used to consider the solvent effect

In this contribution we aim at filling this gap by the use of highlevel computational photochemistry techniques to finely characterize the isomerization process in terms of the nature of the involved excited states, the topology of the PES, and the energy barriers needed to be overcome to finally reach the nonadiabatic crossing regions driving the photochemical process. In this respect, our results will be important to pave the way to the possible exploitation of cyclocurcumin as PDT agents or in photo-triggered drug-delivery strategies. Indeed, cyclocurcumin could destabilize biological lipid membranes through its photoinduced isomerization leading to their apoptosis. The same phenomenon could also be used to force the controlled release of active drugs incorporated in liposomes. However, such a strategy could be reasonably pursued only after a preliminary comprehension of the photochemical pathways of cyclocurcumin, as well as of the influence of complex inhomogeneous environments on such phenomena.

\section{Results and discussion}

The experimental absorption spectrum of cyclocurcumin, most notably reported by Adhikary et $a l, 43$ is characterized by an intense maximum at around $375 \mathrm{~nm}$ that is slightly red-shifted up to $380-385 \mathrm{~nm}$ upon increasing the polarity of the medium. The calculated absorption spectrum, taking into account the vibrational and dynamical effects thanks to the sampling of the ground state conformational space via a Wigner distribution, is reported in Figure 2, while the absorption spectrum for the cis isomer is reported in Supplementary Information. The spectrum in gas phase for the trans isomer calculated using both B3LYP and CAM-B3LYP functionals is reported. Even if the former provides a globally red-shifted spectrum that is closer to the experimental absorption wavelengths $(360 \mathrm{~nm})$, it is not able to recover the correct energy ordering of the excited states leading to an accumulation of partial charge-transfer states in the red tail of the spectrum. For this reason, in the following only the results at the CAM-B3LYP level of theory will be considered. The spectrum of the cis isomer shows a globally decreased oscillator strength, even if a non-negligible overlap with the absorption band of the trans isomer is still observed. The CAM-B3LYP simulated absorption spectrum in the gas phase is characterized by an intense maximum at around 350 $\mathrm{nm}$ followed by an evident tail in the red region extending up to $400-450 \mathrm{~nm}$. Such a band shape is indeed characteristic of the presence of a bright ${ }^{1}\left(\pi, \pi^{*}\right)$ state $\left(S_{2}\right)$ and a darker and lower in energy ${ }^{1}\left(n, \pi^{*}\right)$ state $\left(S_{1}\right)$. The nature of the states is also confirmed by the analysis of the Natural Transition Orbitals (NTOs) describing the first two excited states as reported in Figure 3 , and by the analysis of the oscillator strengths that are of 0.06 and 1.16 for $S_{1}$ and $S_{2}$, respectively. 

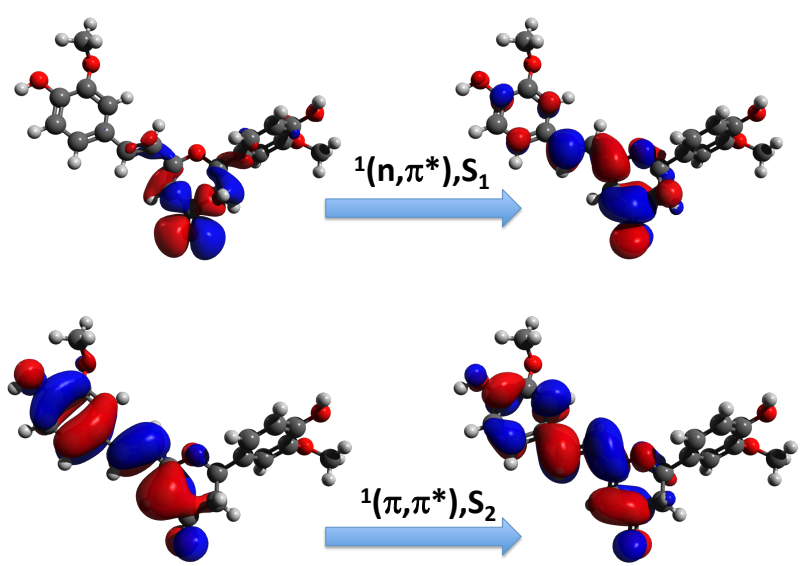

Figure 3) NTOs describing the first two excited states obtained from TD-DFT calculation in gas phase and using the CAM-B3LYP functional.

The calculation of the topological index $\phi_{\mathrm{S}}$, which indicates the charge transfer character of the states, also gives a coherent picture with a value of 0.5 for $S_{1}$, pointing to a moderate charge redistribution, and 0.9 , hence an almost pure locally excited state, for $\mathrm{S}_{2}$. As reported in Figure 2B, and once again coherently with the experimental results, polar solvents induce a slight redshift of the absorption maximum, however without changing the main characteristics of the band shape.

\begin{tabular}{c|c|c|c|c}
\hline Level of theory & $\begin{array}{c}\text { Electronic } \\
\text { state }\end{array}$ & $\begin{array}{c}\text { Franck- } \\
\text { Condon } \\
\text { geometry }\end{array}$ & $\begin{array}{c}{ }^{1}\left(\mathrm{n}, \pi^{*}\right) \\
\text { minimum }\end{array}$ & $\begin{array}{c}{ }^{1}\left(\pi, \pi^{*}\right) \\
\text { minimum }\end{array}$ \\
\hline CASSCF & $\mathrm{S}_{0}$ & 0,00 & 1.36 & 0.97 \\
& $\mathrm{~S}_{1}$ & 4.17 & 2.51 & 3.60 \\
$\mathrm{~S}_{2}$ & 5.40 & 4.74 & 4.40 \\
\hline Multistate & $\mathrm{S}_{0}$ & 0.00 & 1.39 & 0.79 \\
(MS)-CASPT2 & $\mathrm{S}_{1}$ & 3.51 & 3.15 & 3.29 \\
& $\mathrm{~S}_{2}$ & 4.04 & 4.58 & 3.52 \\
\hline Extended & $\mathrm{S}_{0}$ & 0.00 & 1.44 & 0.38 \\
multistate & $\mathrm{S}_{1}$ & 3.69 & 3.39 & 3.65 \\
(XMS)-CASPT2 & $\mathrm{S}_{2}$ & 4.49 & 4.96 & 4.28 \\
\hline TD-DFT & $\mathrm{S}_{0}$ & 0.00 & 0.38 & 0.30 \\
(CAM-B3LYP) & $\mathrm{S}_{1}$ & 3.81 & 3.53 & 3.78 \\
& $\mathrm{~S}_{2}$ & 4.02 & 4.15 & 3.91 \\
\hline
\end{tabular}

Table 1) Relative energies of the ground and the two lowest-lying excited states of cyclocurcumin in gas-phase, calculated at different levels of theory and at different critical points on the PES.

When compared with experimental results ${ }^{43}$, the simulated spectrum appears in good agreement in terms of the maximum absorption wavelengths and of the band shape, even though the CAM-B3LYP functional provides an absorption maximum that is slightly blue-shifted of about $10 \mathrm{~nm}(0.2 \mathrm{eV})$ compared to the experimental results. On the other hand, the experimental solvatochromism is less well reproduced, this fact being most probably due to the use of a continuum model for the solvent and hence neglecting some micro-solvation effects that may become more important for polar and protic media.
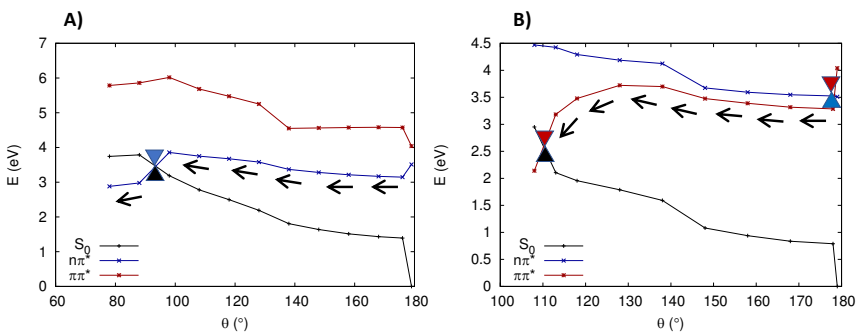

Figure 4) $S_{0} \rightarrow S_{1}(A)$ and $S_{0} \rightarrow S_{2}$ (B) vertical transitions, followed by optimization of the populated ${ }^{1}\left(\mathrm{n}, \pi^{*}\right)(\mathrm{A})$ and $1\left(\pi, \pi^{*}\right)(\mathrm{B})$ excited states. From the respective $\theta$, leading to the photoisomerization, as calculated in gas phase at MS-CASPT2 level.

The calculated values for the fluorescence spectra also compare reasonably well with experimental results. ${ }^{42}$ Indeed, while in gas phase or apolar solvents we obtain an experimental and calculated emission maximum at 420 and $397 \mathrm{~nm}$, respectively, in the case of ethanol, the experimental and calculated maxima are shifted to 500 and $466 \mathrm{~nm}$, respectively. The larger discrepancy with the experimental values is also due to the fact that the computational maxima are obtained as vertical transitions from the $S_{1}$ optimized geometry, hence neglecting vibrational and dynamic effects, usually leading to a red shift. 44,45

As reported in Table 1, the order and the nature of the states provided by CAM-B3LYP is also confirmed by using multiconfigurational complete active space methods. The nature of the states is, once more, confirmed by the analysis of the weight of the molecular orbitals in the CAS expansion (see Supplementary Information). The analysis of the PES for the two low-lying excited states leads to the obtention of two minima that are quite close to the Franck-Condon geometry.

In particular, the ${ }^{1}\left(\pi, \pi^{*}\right)$ minimum is almost totally planar presenting a dihedral angle around the isomerizable doublebond $(\theta)$ of $179^{\circ}$, while the optimization of the ${ }^{1}\left(n, \pi^{*}\right)$ state leads to a slightly more twisted conformation with an equilibrium value of $\theta=176^{\circ}$. The changes in the $\theta$ angle are coupled with the increases of the bond length of the isomerizable double bond - of 0.05 and $0.07 \AA$ for ${ }^{1}\left(n, \pi^{*}\right)$ and ${ }^{1}\left(\pi, \pi^{*}\right)$ minima, respectively. This fact is coherent with the decrease of the double-bond character and the induction of a noticeable bond length alternation. This geometrical deformation already points to the possible triggering of a photoisomerization process, however the presence of a minimum in the PES may also preclude the existence of ultrafast, i.e. sub ps, processes. Interestingly, an accessible $S_{2} / S_{1}$ conical intersection $(\mathrm{Cl})$ is also noticed when irradiating to $S_{2}$, with the diabatic ${ }^{1}\left(\pi, \pi^{*}\right)$ state becoming the first excited state at the corresponding optimized geometry. As a consequence, the participation of both states to the photoisomerization process should be taken into account.

To follow the photoisomerization process in terms of the exploration of the relevant region of the PES of the two lowestenergy excited states, we firstly calculated the relaxed scan along the torsion of the dihedral angle $\theta$ (see Figure 1 ) starting from the respective excited state minima, selecting the 
multistate MS-CASPT2 method in gas phase. Note that, the choice to consider only the value of the dihedral angle to examine the PESs is due to the fact that it is the most relevant (i.e. representative) coordinate in the description of the transcis isomerization. Indeed, as in other well-studied photoswitches (e.g. retinal), $40,46,47$ bond length alternation of single and double bonds is the initial driving force taking place after photon absorption, leading to torsion of the formal double bond implied in the photoisomerization. To discriminate between the participation of the two excited states in the photoprocess, we reported the pathways calculated following the relaxation along the ${ }^{1}\left(n, \pi^{*}\right)$, displayed in Figure $4 A$, and the ${ }^{1}\left(\pi, \pi^{*}\right)$ state, shown in Figure $4 \mathrm{~B}$. In both profiles, one can notice the presence of crossing points between the $S_{0}$ and $S_{1}$ PES, i.e. structures that mediate the excited-state decay via photoisomerization. However, the $\mathrm{S}_{1} / \mathrm{S}_{0} \mathrm{Cl}$ is reached earlier, i.e. at a lower $\theta$ twisting, for the ${ }^{1}\left(\pi, \pi^{*}\right)$ profile, around $115^{\circ}$, whereas the ${ }^{1}\left(n, \pi^{*}\right)$ state requires a more pronounced torsion of about $90^{\circ}$ (Figure 4B). This fact could lead to a decrease in the photoisomerization quantum yield for the ${ }^{1}\left(\pi, \pi^{*}\right)$ profile, due to the occurrence of a considerable number of frustrated hops that would revert to the original trans isomer instead than completing the photoisomerization. On the other hand, the profile to reach the $S_{1} / S_{0} \mathrm{Cl}$ from the ${ }^{1}\left(n, \pi^{*}\right)$ profile is much steeper than the corresponding one calculated for the ${ }^{1}\left(\pi, \pi^{*}\right)$ state, which has a larger excitation energy according to the vertical absorption energies summarised in Table 1 . Indeed, while in the former a barrier of about $0.7 \mathrm{eV}$ needs to be overcome to reach the non-adiabatic point, in the latter the energy penalty is reduced to $c a 0.4 \mathrm{eV}$. Hence, while the photoisomerization from the ${ }^{1}\left(n, \pi^{*}\right)$ state is much less competitive based on energetic considerations, and the system in this state will most likely be trapped around the $S_{1}$ minimum and fluoresce, the photoisomerization pathway is more affordable along the ${ }^{1}\left(\pi, \pi^{*}\right)$ state, even if the $0.4 \mathrm{eV}$ energy barrier found for this profile will globally delay the photoprocess to the picosecond scale.
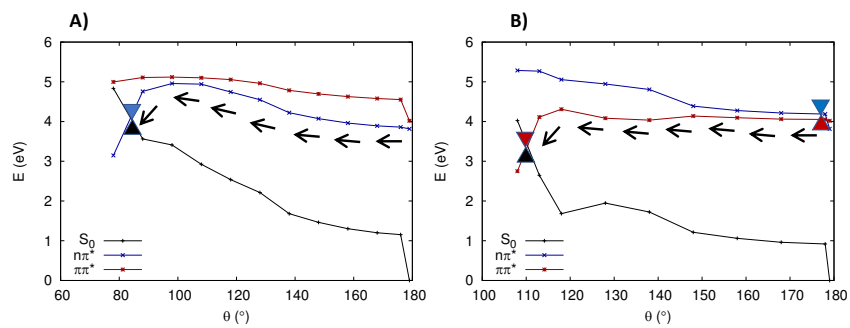

Figure 5) Energy profiles along the torsion of the dihedral angle $\theta$ leading to the photoisomerization, calculated in gas phase at TD-DFT level (using the CAM-B3LYP functional) and following the ${ }^{1}\left(n, \pi^{*}\right)(A)$ and the ${ }^{1}\left(\pi, \pi^{*}\right)$ excited state (B).

Moreover, the moderate distortion of the $\mathrm{S}_{1} / \mathrm{S}_{0} \mathrm{Cl}$ geometry should also decrease the efficiency of the photoprocess.

The profile reported in Figure $4 \mathrm{~B}$ is reminiscent of the peculiar properties of the retinal chromophore when forming the active site of channelrhodopsin ${ }^{47-49}$ and should share the same photochemical characteristics, although a lower excited state barrier in channelrhodopsin can be associated with a faster photoisomerization process ( $\mathrm{S}_{1}$ lifetime of ca. $400 \mathrm{fs}$ ). Note also that the crossing between the ${ }^{1}\left(n, \pi^{*}\right)$ and the ${ }^{1}\left(\pi, \pi^{*}\right)$ states takes place at coordinates extremely close to the FranckCondon region, where the two states are characterized by an extended region of quasi-degeneracy in which the PES evolve almost in parallel. Similar profiles are obtained with the TD-DFT method, as reported in Figure 5. Conversely, the extended multi state XMS-CASPT2 method (see Supplementary Information), while giving the same picture for the ${ }^{1}\left(n, \pi^{*}\right)$ profiles, provides a crossing between the two excited states shifted to smaller $\theta$ values in the case of the ${ }^{1}\left(\pi, \pi^{*}\right)$ pathway.

After having assessed the gas phase photochemistry of cyclocurcumin, the important environmental effects observed experimentally can now be addressed and be related to the influence that the electrostatic field generated by the different solvents on the topology of the PES. As a matter of fact, this influence may lead to state inversion and hence to the tuning of the different accessible photochemical pathways.

In Figure 6 we report the most probable pathway leading to the $\mathrm{S}_{1} / \mathrm{S}_{0} \mathrm{Cl}$ structures that trigger the photoisomerization, calculated in non-polar (cyclohexane), polar (acetonitrile), and protic (ethanol and water) solvents. As expected, the behaviour of cyclocurcumin is strongly modified depending on the molecular surroundings. In the non-polar medium at FranckCondon region, both ${ }^{1}\left(n, \pi^{*}\right)$ and ${ }^{1}\left(\pi, \pi^{*}\right)$ states could be initially populated, leading in any case to a rather steep PES leading first to a ${ }^{1}\left(n, \pi^{*}\right) /{ }^{1}\left(\pi, \pi^{*}\right)$ crossing (at around $\theta=140^{\circ}$ ) and subsequently to the $\mathrm{S}_{1} / \mathrm{S}_{0} \mathrm{Cl}$ allowing photoisomerization. In contrast, for polar and protic solvents, the optically brighter ${ }^{1}\left(\pi, \pi^{*}\right)$ state is stabilized in energy, becoming the $S_{1}$ state at Franck-Condon region, largely increasing the probability to be initially populated when irradiating the compound. Moreover, the Franck-Condon and the $\mathrm{S}_{1} / \mathrm{S}_{0} \mathrm{Cl}$ structures are connected through globally barrierless or almost barrierless PESs, thus leading to photoisomerization. As a consequence, the ${ }^{1}\left(n, \pi^{*}\right)$ state is destabilized in polar solvents precluding any ${ }^{1}\left(\mathrm{n}, \pi^{*}\right) /{ }^{1}\left(\pi, \pi^{*}\right)$ crossing.
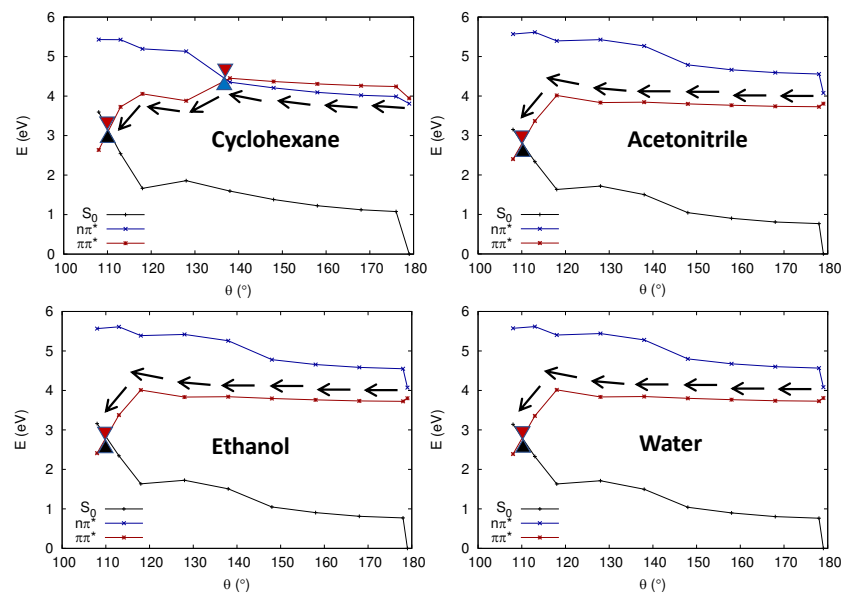

Figure 6) Energy profiles along the torsion of the dihedral angle $\theta$ calculated at TDDFT level in presenc e of different solvents 
This means that, in polar and protic solvents, cyclocurcumin photophysics can be considered as a 2-(electronic) states system. In stark contrast, in non-polar environments cyclocurcumin is a 3-states system, mostly trapped in the region of the ${ }^{1}\left(n, \pi^{*}\right)$ minimum and hence giving rise to fluorescence. On the contrary, polar media favours photoisomerization over luminescence, and the fluorescence quantum yield is significantly decreased. This picture is in perfect agreement with the experimental results of Adhikary et al., ${ }^{43}$ who have clearly shown fluorescence quenching when increasing the solvent polarity, whereas the radiative decay is active in cyclohexane. Hence, it is clear that polar solvents will activate non-radiative decay pathways leading to trans-to-cis isomerization, consistently diminishing the lifetime of the excited states and preventing luminescence. In addition, since the isomerization is proceeding through a rather pronounced spatial movement involving large molecular fragments, the viscosity of the medium is supposed to play a fundamental role as well.

Our results offer a clear electronic-based picture of the characteristic behaviour of cyclocurcumin. As an important consequence, the possible modulation of the photochemical properties by the environment could qualify cyclocurcumin as a bifunctional photoswitch and could potentially be exploited for therapeutic purposes in light-assisted chemotherapy. Indeed, in the complex and inhomogeneous environment, offered for instance by lipid bilayers, it is expected that the delicate equilibrium between luminescence and photoisomerization will become even more complex and could also depend on the position of cyclocurcumin with respect to the membrane axis. However, the coexistence of isomerization and fluorescence can be exploited to combine diagnosis and treatment, as in a theranostic-like approach. Furthermore, preliminary TD-DFT calculations performed at the Franck-Condon geometry have shown that cyclocurcumin has a non-negligible two-photon absorption (TPA), allowing to reach the infrared region. Indeed, the calculated cross section for TPA, in gas phase, amounts to $14 \mathrm{GM}$, a value that is relatively high for simple organic molecules, offering attractive therapeutic possibilities for this compound. Even though the total elucidation of the non-linear optical properties of cyclocurcumin and their dependence on the environment is well beyond the scope of the present contribution, the calculated values of the TPA cross-section and related wavelengths, together with its inherent photochemistry, definitively suggest the possible use of cyclocurcumin as a TPA active drug that may be operative in the therapeutic window, also exploiting a much higher spatial resolution thanks to the non-linear nature of TPA.

\section{Conclusions}

By using molecular modelling techniques, and in particular by a proper exploration of the nature and topology of the excited states of cyclocurcumin, including the exploration of the PES along relevant coordinates, we have clearly demonstrated its capability to act as a photoisomerizable switch. In particular, we have shown the relevant role played by the two lowest excited states, ${ }^{1}\left(\mathrm{n}, \pi^{*}\right)$ and ${ }^{1}\left(\pi, \pi^{*}\right)$, and how the photoisomerization will be favoured while proceeding on the ${ }^{1}\left(\pi, \pi^{*}\right)$ PES. In turn, we have also shown how the photoresponse is strongly modified by the molecular environment: polar solvents favour the population of the ${ }^{1}\left(\pi, \pi^{*}\right)$ state, hence leading to a flatter PES giving access to the $\mathrm{S}_{1} / \mathrm{S}_{0} \quad \mathrm{Cl}$ structures that trigger photoisomerization, while non-polar solvents stabilize the ${ }^{1}\left(\mathrm{n}, \pi^{*}\right)$ state hampering the access to the crossing region with the ground state. As a consequence, while in polar solvents cyclocurcumin is expected to photoisomerize, albeit not at an ultrafast time scale, in non-polar environments the fluorescence will be dominant. This bifunctional nature of cyclocurcumin could lead to fascinating applications in lightassisted chemotherapy and in theranostics, also thanks to the previewed relatively high TPA cross section that could allow its activation via infrared light, hence covering the biological therapeutic window. All in all, our results clearly point to the possible use of cyclocurcumin for biotechnological or, in the longer time scale, medical purposes in particular exploiting its photoisomerization. Cyclocurcumin can offer an alternative photochemical pathway compared to other turmeric extracts, such as curcumin, whose light-activated mechanism of action is dominated by intersystem crossing and hence the activation of molecular oxygen through Type II photosensitization. Hence, cyclocurcumin could be efficiently used in conditions of hypoxic solid tumours that can be resistant to conventional PDT. However, the present results also offer some important suggestions on possible rational drug design strategies that should be pursued to maximize cyclocurcumin efficiency, also going above the simple, yet crucial, increase of its bioavailability. In particular, one can imagine to selectively stabilize the $\pi \pi^{*}$ state over the $n \pi^{*}$ to increase the photoisomerization quantum yield.

As an alternative application, the environmental dependence of photoisomerization and fluorescence quantum yield with the polarity of the medium points to the potential application of cyclocurcumin derivates as chemosensors, detecting in real time polarity changes in micro-environments. ${ }^{50}$

In the future, we plan to carefully investigate the properties of cyclocurcumin and its derivatives for their inclusion in lipid bilayers, mimicking liposomes or biological membranes, using both experimental time-resolved spectroscopy techniques and hybrid quantum mechanics/molecular mechanics simulations to assess the effects of inhomogeneous environments on its photochemical properties. In the same way, the influence of the dynamical and vibrational degrees of freedom and of the complex surroundings on TPA will also be pursued, including the eventual sensitization of cyclocurcumin with specific TPA antennas to increase the relevance of this feature.

\section{Computational Methodology}

Trans- and cis-cyclocurcumin ground state minimum structures were located by Density Functional Theory (DFT), by applying the B3LYP functional. ${ }^{51}$ The absorption spectrum of both isomers was calculated by using the Time Dependent flavour of DFT (TD-DFT), using B3LYP51 and CAM-B3LYP52 functionals. 
More in detail, the ground state phase space was sampled around the Franck-Condon structure via a Wigner distribution, using the NewtonX $\operatorname{code}^{53}$ to generate 100 structures. Excited states were computed for each structure through single point calculations, finally convoluting all Gaussian functions resulting from all transition energies and oscillator strengths (full width at half maximum $=0.2$ $\mathrm{eV})$. This approach to simulate the absorption spectrum of organic chromophores was proven to be adequate by some of the authors. ${ }^{44,54-57}$

To properly select a convenient active space for multi-configurational methods, the absorption spectrum was calculated also for the B3LYP Franck-Condon structure at MS-CASPT2//SA(4)-CASSCF level,58,59 i.e. a first Complete Active Space Self-Consistent Field calculation was performed including 4 averaged states (from $S_{0}$ to $S_{3}$ ), followed by a multistate calculation applying $2^{\text {nd }}$ order perturbation theory to the CASSCF wavefunction. As shown in Figure S3 of the Supplementary Information, three active states were tested, in all cases including a n orbital and several $\pi$ and $\pi^{*}$ orbitals: 10 electrons in 9 orbitals $(10,9), 12$ electrons in 11 orbitals $(12,11)$, and 14 electrons in 13 orbitals $(14,13)$. All active spaces predict a bright $S_{0} \rightarrow S_{2}$ vertical transition, whereas the $S_{0} \rightarrow S_{1}$ is notably dark. This finding is coherent with the fact that in gas phase the $S_{0} \rightarrow S_{1}$ transition is of a ${ }^{1}\left(n, \pi^{*}\right)$ nature, while $S_{0} \rightarrow S_{2}$ is of ${ }^{1}\left(\pi, \pi^{*}\right)$ character.

Hence, as a compromise between accuracy and required computational cost, the photochemistry of cyclocurcumin was studied with the $(10,9)$ active space. In particular, the $S_{1}\left(n \pi^{*}\right)$ and $S_{2}$ $\left(\pi \pi^{*}\right)$ states identified at the Franck-Condon region were optimized with the CASSCF method, reaching the respective minima whose diabatic nature was confirmed by analysing the respective molecular orbitals. Starting from these structures, we performed relaxed scans along the dihedral angle $\theta$ optimizing both $S_{1}$ and $S_{2}$ states. In addition to multiconfigurational method TD-DFT calculations were also performed.

On top of all CASSCF optimized structures, single point energy calculations were performed at different level of theory in order to compute the necessary dynamic electron correlation: MS-CASPT2, extended (X)MS-CASPT2,60 and CAM-B3LYP (with and without spinflip), in gas phase. Considering the correct description given by CAMB3LYP, solvent effects were included at this level of theory: cyclohexane, acetonitrile, ethanol and water were considered by applying the equilibrium state-specific Polarizable Continuum Model (PCM) within the integral equation formalism variant (IEFPCM). ${ }^{61,62}$ Although TD-DFT is problematic in correctly describing $\mathrm{S}_{1} / \mathrm{S}_{0} \mathrm{Cls}$ due to the lack of multiconfigurational character for the ground state, 63 it has to be noted that our TD-DFT results are obtained on top of CASSCF optimized geometries (i.e. TD-DFT//CASSCF approach), and that the gas phase PES obtained at TD-DFT and CASPT2 level agrees considerably, pointing to the robustness of our approach. To further confirm this point, we have also repeated the calculation of the gas phase PES using a spin-flip TD-DFT63-66//CASSCF approach (Supplementary Information) that also provides a coherent picture with both multiconfigurational and TD-DFT results.

For all multi-configurational calculations, the 6-31G* basis set was adopted, while the $6-311+G^{*}$ basis set was used for DFT and TD-DFT calculations. Spin-flip TD-DFT calculations were performed with the TZP basis set.

In the case of MS- and XMS-CASPT2 calculations, no IPEA was applied, while an imaginary shift of 0.2 a.u. was considered to avoid intruder states. Both MS- and XMS-CASPT2 methods were applied, since the latter is known to better perform in regions of energy degeneracy, ${ }^{60}$ while the former is more suitable to treat electronic states far in energy. Hence, the use of both methods was considered necessary to give an average correct description.

All CASSCF and CASPT2 calculations were run with the OpenMolcas suite of programs, ${ }^{67,68}$ while DFT and TD-DFT calculations were performed with the Gaussian09 software. ${ }^{69}$ TPA cross-section have been determined at TD-DFT level using the Dalton package ${ }^{70,71}$ and a protocol already successfully used for different organic dyes..$^{54,72}$ Spin-flip calculations were performed with ADF2018 modelling suite. ${ }^{73}$

The charge transfer character of the excited states was evaluated at TD-DFT level by applying the topological index $\phi_{\mathrm{s}}{ }^{74-76}$ which tends to 1 (0) for locally (charge transfer) excited states, as it is an estimation of the overlap between occupied and virtual Natural Transition Orbitals (NTOs) ${ }^{77}$ involved in the electronic transition.

\section{Conflicts of interest}

There are no conflicts to declare.

\section{Acknowledgements}

Support from the Universite de Lorraine and the French CNRS is gratefully acknowledged. DFT and TD-DFT calculations were performed on the LPCT local computing cluster and on the computing centre of the Universite de Lorraine (Explor) in the framework of the project "Dancing Under the Light". CASSCF and CASPT2 calculations were performed on the local computing cluster of the Physical Chemistry Unit of the Universidad de Alcalá. A.F.-M. is grateful to the Generalitat Valenciana and the European Social Fund for the postdoctoral contract (APOSTD/2019/149).

\section{References}

(1) Sasikumar, B. Turmeric. In Handbook of Herbs and Spices: Second Edition; Elsevier Inc., 2012; Vol. 1, pp 526-546.

(2) Cas, M. D.; Ghidoni, R. Dietary Curcumin: Correlation between Bioavailability and Health Potential. Nutrients 2019, 11 (9), 2147.

(3) Priyadarsini, K. I. The Chemistry of Curcumin: From Extraction to Therapeutic Agent. Molecules 2014, 19 (12), 20091-20112.

(4) Kuptniratsaikul, V.; Dajpratham, P.; Taechaarpornkul, W.; Buntragulpoontawee, M.; Lukkanapichonchut, P.; Chootip, C.; Saengsuwan, J.; Tantayakom, K.; Laongpech, S. Efficacy and Safety of Curcuma Domestica Extracts Compared with Ibuprofen in Patients with Knee Osteoarthritis: A 
Multicenter Study. Clin. Interv. Aging 2014, 9, 451-458.

(5) Mazzolani, F.; Togni, S. Oral Administration of a CurcuminPhospholipid Delivery System for the Treatment of Central Serous Chorioretinopathy: A 12-Month Follow-up Study. Clin. Ophthalmol. 2013, 7, 939-945.

(6) Aggarwal, B. B.; Harikumar, K. B. Potential Therapeutic Effects of Curcumin, the Anti-Inflammatory Agent, against Neurodegenerative, Cardiovascular, Pulmonary, Metabolic, Autoimmune and Neoplastic Diseases. Int. J. Biochem. Cell Biol. 2009, 41 (1), 40-59.

(7) Reddy, R. C.; Vatsala, P. G.; Keshamouni, V. G.; Padmanaban, G.; Rangarajan, P. N. Curcumin for Malaria Therapy. Biochem. Biophys. Res. Commun. 2005, 326 (2), 472-474.

(8) Wright, L.; Frye, J.; Gorti, B.; Timmermann, B.; Funk, J. Bioactivity of Turmeric-Derived Curcuminoids and Related Metabolites in Breast Cancer. Curr. Pharm. Des. 2013, 19 (34), 6218-6225.

(9) Vera-Ramirez, L.; Pérez-Lopez, P.; Varela-Lopez, A.; Ramirez-Tortosa, M.; Battino, M.; Quiles, J. L. Curcumin and Liver Disease. BioFactors 2013, 39 (1), 88-100.

(10) Hewlings, S.; Kalman, D. Curcumin: A Review of Its' Effects on Human Health. Foods 2017, 6 (10), 92.

(11) Ormond, A. B.; Freeman, H. S. Dye Sensitizers for Photodynamic Therapy. Materials (Basel). 2013, 6 (3), 817840.

(12) Penha, C. B.; Bonin, E.; da Silva, A. F.; Hioka, N.; Zanqueta, É. B.; Nakamura, T. U.; de Abreu Filho, B. A.; CampanerutSá, P. A. Z.; Mikcha, J. M. G. Photodynamic Inactivation of Foodborne and Food Spoilage Bacteria by Curcumin. LWT Food Sci. Technol. 2017, 76, 198-202.

(13) Brovko, L.; Anany, H.; Bayoumi, M.; Giang, K.; Kunkel, E.; Lim, E.; Naboka, O.; Rahman, S.; Li, J.; Filipe, C. D. M.; et al. Antimicrobial Light-Activated Materials: Towards Application for Food and Environmental Safety. J. Appl. Microbiol. 2014, 117 (5), 1260-1266.

(14) Mangolim, C. S.; Moriwaki, C.; Nogueira, A. C.; Sato, F.; Baesso, M. L.; Neto, A. M.; Matioli, G. Curcumin- $\beta$ Cyclodextrin Inclusion Complex: Stability, Solubility, Characterisation by FT-IR, FT-Raman, X-Ray Diffraction and Photoacoustic Spectroscopy, and Food Application. Food Chem. 2014, 153, 361-370.

(15) Cui, J.; Yu, B.; Zhao, Y.; Zhu, W.; Li, H.; Lou, H.; Zhai, G. Enhancement of Oral Absorption of Curcumin by SelfMicroemulsifying Drug Delivery Systems. Int. J. Pharm. 2009, 371 (1-2), 148-155.

(16) Sun, D.; Zhuang, X.; Xiang, X.; Liu, Y.; Zhang, S.; Liu, C.; Barnes, S.; Grizzle, W.; Miller, D.; Zhang, H. G. A Novel Nanoparticle Drug Delivery System: The Anti-Inflammatory Activity of Curcumin Is Enhanced When Encapsulated in Exosomes. Mol. Ther. 2010, 18 (9), 1606-1614.

(17) Anitha, A.; Maya, S.; Deepa, N.; Chennazhi, K. P.; Nair, S. V.; Tamura, H.; Jayakumar, R. Efficient Water Soluble OCarboxymethyl Chitosan Nanocarrier for the Delivery of Curcumin to Cancer Cells. Carbohydr. Polym. 2011, 83 (2), 452-461.

(18) Bansal, S. S.; Goel, M.; Aqil, F.; Vadhanam, M. V.; Gupta, R.
C. Advanced Drug Delivery Systems of Curcumin for Cancer Chemoprevention. Cancer Prevention Research. August 2011, pp 1158-1171.

(19) Gupta, S. C.; Patchva, S.; Aggarwal, B. B. Therapeutic Roles of Curcumin: Lessons Learned from Clinical Trials. AAPS Journal. January 2013, pp 195-218.

(20) Wang, H.; Sui, H.; Zheng, Y.; Jiang, Y.; Shi, Y.; Liang, J.; Zhao, L. Curcumin-Primed Exosomes Potently Ameliorate Cognitive Function in AD Mice by Inhibiting Hyperphosphorylation of the Tau Protein through the AKT/GSK-3ß Pathway. Nanoscale 2019, 11 (15), 74817496.

(21) Girardon, M.; Parant, S.; Monari, A.; Dehez, F.; Chipot, C.; Rogalska, E.; Canilho, N.; Pasc, A. Triggering Tautomerization of Curcumin by Confinement into Liposomes. ChemPhotoChem 2019, 3 (10), 1034-1041. Kim, K.; Kim, J. J.; Jung, Y.; Noh, J. Y.; Syed, A. S.; Kim, C. Y.; Lee, M. Y.; Lim, K. M.; Bae, O. N.; Chung, J. H.

Cyclocurcumin, an Antivasoconstrictive Constituent of Curcuma Longa (Turmeric). J. Nat. Prod. 2017, 80 (1), 196200.

(23) Fu, M.; Chen, L.; Zhang, L.; Yu, X.; Yang, Q. Cyclocurcumin, a Curcumin Derivative, Exhibitsimmune-Modulating Ability and Is a Potential Compound for the Treatment of Rheumatoid Arthritisas Predicted by the MM-PBSA Method. Int. J. Mol. Med. 2017, 39 (5), 1164-1172. Chakraborty, S.; Karmenyan, A.; Tsai, J. W.; Chiou, A. Inhibitory Effects of Curcumin and Cyclocurcumin in 1Methyl-4-Phenylpyridinium (MPP+) Induced Neurotoxicity in Differentiated PC12 Cells. Sci. Rep. 2017, 7 (1), 16977. Ravindran, J.; Prasad, S.; Aggarwal, B. B. Curcumin and Cancer Cells: How Many Ways Can Curry Kill Tumor Cells Selectively? AAPS J. 2009, 11 (3), 495-510.

(26) Hubbard, R.; Wald, G. Cis-Trans Isomers of Vitamin A and Retinene in the Rhodopsin System. J. Gen. Physiol. 1952, 36 (2), 269-315.

(27) Wald, G. Molecular Basis of Visual Excitation. Science. American Association for the Advancement of Science October 1968, pp 230-239.

(28) Altoè, P.; Cembran, A.; Olivucci, M.; Garavelli, M. Aborted Double Bicycle-Pedal Isomerization with Hydrogen Bond Breaking Is the Primary Event of Bacteriorhodopsin Proton Pumping. Proc. Natl. Acad. Sci. U. S. A. 2010, 107 (47), 20172-20177.

(29) Briand, J.; Bräm, O.; Réhault, J.; Léonard, J.; Cannizzo, A.; Chergui, M.; Zanirato, V.; Olivucci, M.; Helbing, J.; Haacke, S. Coherent Ultrafast Torsional Motion and Isomerization of a Biomimetic Dipolar Photoswitch. Phys. Chem. Chem. Phys. 2010, 12 (13), 3178-3187.

(30) González-Luque, R.; Garavelli, M.; Bernardi, F.; Merchán, M.; Robb, M. A.; Olivucci, M. Computational Evidence in Favor of a Two-State, Two-Mode Model of the Retinal Chromophore Photoisomerization. Proc. Natl. Acad. Sci. U. S. A. 2000, 97 (17), 9379-9384.

(31) Tomasello, G.; Gloria, O. G.; Altoè, P.; Stenta, M.; Luis, S. A.; Merchán, M.; Orlandi, G.; Bottoni, A.; Garavelli, M. Electrostatic Control of the Photoisomerization Efficiency 
and Optical Properties in Visual Pigments: On the Role of Counterion Quenching. J. Am. Chem. Soc. 2009, 131 (14), 5172-5186.

(32) Aman, R.; Schieber, A.; Carle, R. Effects of Heating and Illumination on Trans-Cis Isomerization and Degradation of $\beta$-Carotene and Lutein in Isolated Spinach Chloroplasts. J. Agric. Food Chem. 2005, 53 (24), 9512-9518.

(33) Aleotti, F.; Soprani, L.; Nenov, A.; Berardi, R.; Arcioni, A.; Zannoni, C.; Garavelli, M. Multidimensional Potential Energy Surfaces Resolved at the RASPT2 Level for Accurate Photoinduced Isomerization Dynamics of Azobenzene. J. Chem. Theory Comput. 2019, 10.1021/acs.jctc.9b00561.

(34) Tiberio, G.; Muccioli, L.; Berardi, R.; Zannoni, C. How Does the Trans-Cis Photoisomerization of Azobenzene Take Place in Organic Solvents? ChemPhysChem 2010, 11 (5), 1018-1028.

(35) Bandara, H. M. D.; Burdette, S. C. Photoisomerization in Different Classes of Azobenzene. Chemical Society Reviews. March 7, 2012, pp 1809-1825.

(36) Waldeck, D. H. Photoisomerization Dynamics of Stilbenes. Chem. Rev. 1991, 91 (3), 415-436.

(37) Bylina, A.; Grabowski, Z. R. Photo-Isomerization and the Triplet State of Stilbene. Trans. Faraday Soc. 1969, 65, 458-463.

(38) Tomasello, G.; Garavelli, M.; Orlandi, G. Tracking the Stilbene Photoisomerization in the S1 State Using RASSCF. Phys. Chem. Chem. Phys. 2013, 15 (45), 19763-19773.

(39) García-Iriepa, C.; Marazzi, M.; Frutos, L. M.; Sampedro, D. E/Z Photochemical Switches: Syntheses, Properties and Applications. RSC Adv. 2013, 3 (18), 6241-6266.

(40) Marazzi, M.; Garcia-Iriepa, C. Retinal Inspired Photoswitches. In Photoisomerization causes, behavior, and effects; Sampedro, D., Ed.; Nova Science Publisher, 2019; pp 189-238.

(41) Garcia-Iriepa, C.; Gueye, M.; Leonard, J.; Martinez-Lopez, D.; Campos, P. J.; Frutos, L. M.; Sampedro, D.; Marazzi, M. A Biomimetic Molecular Switch at Work: Coupling Photoisomerization Dynamics to Peptide Structural Rearrangement. Phys. Chem. Chem. Phys. 2016, 18 (9), 6742-6753.

(42) Sampedro, D.; Migani, A.; Pepi, A.; Busi, E.; Basosi, R.; Latterini, L.; Elisei, F.; Fusi, S.; Ponticelli, F.; Zanirato, V.; et al. Design and Photochemical Characterization of a Biomimetic Light-Driven Z/E Switcher. J. Am. Chem. Soc. 2004, 126 (30), 9349-9359.

(43) Adhikary, R.; Barnes, C. A.; Trampel, R. L.; Wallace, S. J.; Kee, T. W.; Petrich, J. W. Photoinduced Trans-to-Cis Isomerization of Cyclocurcumin. J. Phys. Chem. B 2011, 115 (36), 10707-10714.

(44) Gattuso, H.; Monari, A.; Marazzi, M. Photophysics of Chlorin E6: From One- and Two-Photon Absorption to Fluorescence and Phosphorescence. RSC Adv. 2017, 7 (18), 10992-10999.

(45) Moron, V.; Marazzi, M.; Wanko, M. Far Red Fluorescent Proteins: Where Is the Limit of the Acylimine Chromophore? J. Chem. Theory Comput. 2019, 15 (7), 4228-4240.
(46) Guo, Y.; Wolff, F. E.; Schapiro, I.; Elstner, M.; Marazzi, M. Different Hydrogen Bonding Environments of the Retinal Protonated Schiff Base Control the Photoisomerization in Channelrhodopsin-2. Phys. Chem. Chem. Phys. 2018, 20 (43), 27501-27509.

(47) Hontani, Y.; Marazzi, M.; Stehfest, K.; Mathes, T.; Van Stokkum, I. H. M.; Elstner, M.; Hegemann, P.; Kennis, J. T. $M$. Reaction Dynamics of the Chimeric Channelrhodopsin. Sci. Rep. 2017, 7 (1), 7217.

(48) Guo, Y.; Beyle, F. E.; Bold, B. M.; Watanabe, H. C.; Koslowski, A.; Thiel, W.; Hegemann, P.; Marazzi, M.; Elstner, M. Active Site Structure and Absorption Spectrum of Channelrhodopsin-2 Wild-Type and C128T Mutant. Chem. Sci. 2016, 7 (6), 3879-3891.

(49) Welke, K.; Watanabe, H. C.; Wolter, T.; Gaus, M.; Elstner, M. QM/MM Simulations of Vibrational Spectra of Bacteriorhodopsin and Channelrhodopsin-2. Phys. Chem. Chem. Phys. 2013, 15 (18), 6651.

(50) Yang, Z.; Cao, J.; He, Y.; Yang, J. H.; Kim, T.; Peng, X.; Kim, J. S. Macro-/Micro-Environment-Sensitive Chemosensing and Biological Imaging. Chemical Society Reviews. Royal Society of Chemistry July 7, 2014, pp 4563-4601.

(51) Becke, A. B3LYP. J. Chem. Phys. 1993, 98, 5648-5652.

(52) Yanai, T.; Tew, D. P.; Handy, N. C. A New Hybrid ExchangeCorrelation Functional Using the Coulomb-Attenuating Method (CAM-B3LYP). Chem. Phys. Lett. 2004, 393 (1-3), 51-57.

(53) Barbatti, M.; Ruckenbauer, M.; Plasser, F.; Pittner, J.; Granucci, G.; Persico, M.; Lischka, H. Newton-X: A SurfaceHopping Program for Nonadiabatic Molecular Dynamics. Wiley Interdiscip. Rev. Comput. Mol. Sci. 2014, 4 (1), 26-33. Gattuso, H.; Dumont, E.; Marazzi, M.; Monari, A. TwoPhoton-Absorption DNA Sensitization via Solvated Electrons Production: Unraveling the Photochemical Pathways by Molecular Modeling and Simulation. Phys. Chem. Chem. Phys. 2016, 18 (27), 18598-18606.

(55) Assfeld, X.; Monari, A.; Marazzi, M.; Gattuso, H. SteadyState Linear and Non-Linear Optical Spectroscopy of Organic Chromophores and Bio-Macromolecules. Front. Chem. 2018, 6, 86.

(56) Turan, H.; Eken, Y.; Marazzi, M.; Pastore, M.; Aviyente, V.; Monari, A. Assessing One- and Two-Photon Optical Properties of Boron Containing Arenes. J. Phys. Chem. C 120 (32), 17916-17926.

(57) Koca, B.; Hamuryudan, E.; Catak, S.; Erdogmus, A.; Monari, A.; Aviyente, V. Exploring the Photophysics of Polyfluorinated Phthalocyanine Derivatives as Potential Theranostic Agents. J. Phys. Chem. C 2019.

(58) Andersson, K.; Malmqvist, P.-A.; Roos, B. O.; A. J. Sadlej; Wolinski, K. Second-Order Perturbation Theory with a CASSCF Reference Function. J. Phys. Chem. 1990, 94, 54835488.

(59) Andersson, K.; Malmqvist, P.-A.; Roos, B. O. Second-Order Perturbation Theory with a Complete Active Space SelfConsistent Field Reference Function. J. Chem. Phys. 1992, 96, 1218.

(60) Shiozaki, T.; Gyroffy, W.; Celani, P.; Werner, H. J. Extended 
Multi-State Complete Active Space Second-Order Perturbation Theory: Energy and Nuclear Gradients. J. Chem. Phys. 2011, 135 (8), 081106.

(61) Mennucci, B. Polarizable Continuum Model. Wiley Interdiscip. Rev. Comput. Mol. Sci. 2012, 2 (3), 386-404.

(62) Tomasi, J.; Mennucci, B.; Cammi, R. Quantum Mechanical Continuum Solvation Models. Chem. Rev. 2005, 105 (8), 2999-3093.

(63) Levine, B. G.; Ko, C.; Quenneville, J.; Martínez, T. J. Conical Intersections and Double Excitations in Time-Dependent Density Functional Theory. Mol. Phys. 2006, 104 (5-7), 1039-1051.

(64) Gozem, S.; Melaccio, F.; Valentini, A.; Filatov, M.; HuixRotllant, M.; Ferré, N.; Frutos, L. M.; Angeli, C.; Krylov, A. I.; Granovsky, A. A.; et al. Shape of Multireference, Equationof-Motion Coupled-Cluster, and Density Functional Theory Potential Energy Surfaces at a Conical Intersection. J. Chem. Theory Comput. 2014, 10 (8), 3074-3084.

(65) Zhang, X.; Herbert, J. M. Analytic Derivative Couplings for Spin-Flip Configuration Interaction Singles and Spin-Flip Time-Dependent Density Functional Theory. J. Chem. Phys. 2014, 141 (6).

(66) Minezawa, N.; Gordon, M. S. Optimizing Conical Intersections by Spin-Flip Density Functional Theory: Application to Ethylene. J. Phys. Chem. A 2009, 113 (46), 12749-12753.

(67) Aquilante, F.; Autschbach, J.; Carlson, R. K. K.; Chibotaru, L. F. F.; Delcey, M. G. C.; De Vico, L.; Fdez. Galván, I.; Ferré, N.; Frutos, L. M. M.; Gagliardi, L.; et al. Molcas 8: New Capabilities for Multiconfigurational Quantum Chemical Calculations across the Periodic Table. J. Comput. Chem. 2015, 37 (5), doi: 10.1002/jcc.24221.

(68) Fdez. Galván, I.; Vacher, M.; Alavi, A.; Angeli, C.; Aquilante, F.; Autschbach, J.; Bao, J. J.; Bokarev, S. I.; Bogdanov, N. A.; Carlson, R. K.; et al. OpenMolcas: From Source Code to Insight. J. Chem. Theory Comput. 2019, 15 (11), doi: 10.1021/acs.jctc.9b00532.

(69) Frisch, M. J.; Trucks, G. W.; Schlegel, H. B.; Scuseria, G. E.; Robb, M. A.; Cheeseman, J. R.; Scalmani, G.; Barone, V.; Mennucci, B.; Petersson, G. A.; et al. Gaussian09 Revision D.01, Gaussian Inc. Wallingford CT. Gaussian 09 Revision D.01. 2010, p Gaussian Inc., Wallingford CT.

(70) Dalton, a molecular electronic structure program. Release Dalton2016.A, see http://daltonprogram.org.

(71) Aidas, K.; Angeli, C.; Bak, K. L.; Bakken, V.; Bast, R.; Boman, L.; Christiansen, O.; Cimiraglia, R.; Coriani, S.; Dahle, P.; et al. The Dalton Quantum Chemistry Program System. Wiley Interdiscip. Rev. Comput. Mol. Sci. 2014, 4 (3), 269-284.

(72) Turan, H. T.; Eken, Y.; Marazzi, M.; Pastore, M.; Aviyente, V.; Monari, A. Assessing One- and Two-Photon Optical Properties of Boron Containing Arenes. J. Phys. Chem. C 2016, 120 (32).

(73) te Velde, G.; Bickelhaupt, F. M.; Baerends, E. J.; Fonseca Guerra, C.; van Gisbergen, S. J. A.; Snijders, J. G.; Ziegler, T. Chemistry with ADF. J. Comput. Chem. 2001, 22 (9), 931967.

(74) Etienne, T.; Assfeld, X.; Monari, A. Toward a Quantitative
Assessment of Electronic Transitions" Charge-Transfer Character. J. Chem. Theory Comput. 2014, 10 (9), 38963905.

(75) Etienne, T.; Assfeld, X.; Monari, A. New Insight into the Topology of Excited States through Detachment/Attachment Density Matrices-Based Centroids of Charge. J. Chem. Theory Comput. 2014, 10 (9), 3906-3914.

(76) Pastore, M.; Assfeld, X.; Mosconi, E.; Monari, A.; Etienne, T. Unveiling the Nature of Post-Linear Response Z-Vector Method for Time-Dependent Density Functional Theory. J. Chem. Phys. 2017, 147 (2), 024108.

(77) Martin, R. L. Natural Transition Orbitals. J. Chem. Phys. 2003, 118 (11), 4775-4777. 


\section{Graphical Abstract}

Cyclocurcumin

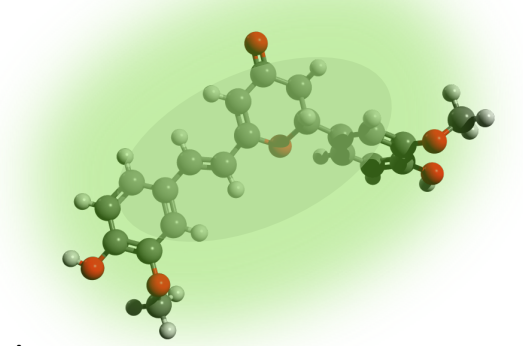

Fluorescence vs Isomerization

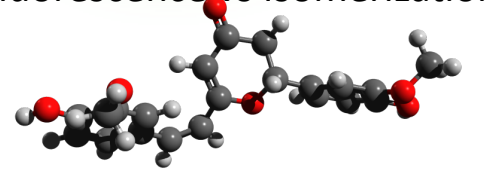

